\title{
Robust Gag-specific T cell responses characterize viremia control in HIV-2 infection
}

\author{
Aleksandra Leligdowicz,1,2 Louis-Marie Yindom, ${ }^{1}$ Clayton Onyango, ${ }^{1}$ Ramu Sarge-Njie, ${ }^{1}$ \\ Abraham Alabi, ${ }^{1}$ Matthew Cotten, ${ }^{1}$ Tim Vincent,, ${ }^{1,3}$ Carlos da Costa, ${ }^{3}$ Peter Aaby, ${ }^{3}$ Assan Jaye, ${ }^{1}$ \\ Tao Dong, ${ }^{2}$ Andrew McMichael, ${ }^{2}$ Hilton Whittle, ${ }^{1}$ and Sarah Rowland-Jones ${ }^{1,2}$ \\ ${ }^{1}$ Medical Research Council Laboratories, Fajara, Republic of The Gambia. ${ }^{2}$ Weatherall Institute of Molecular Medicine, Medical Research Council \\ Human Immunology Unit, John Radcliffe Hospital, Oxford, United Kingdom. 3Projecto Saude Bandim, Bissau, Republic of Guinea-Bissau.
}

\begin{abstract}
HIV-2 infection in the majority of infected subjects follows an attenuated disease course that distinguishes it from infection with HIV-1. Antigen-specific $\mathrm{T}$ cells are pivotal in the management of chronic viral infections but are not sufficient to control viral replication in HIV-1-positive subjects, and their function in HIV-2 infection is not fully established. In a community-based cohort of HIV-2 long-term nonprogressors in rural Guinea-Bissau, we performed what we believe is the first comprehensive analysis of HIV-2-specific immune responses. We demonstrate that Gag is the most immunogenic protein. The magnitude of the IFN- $\gamma$ immune response to the HIV-2 proteome was inversely correlated with HIV-2 viremia, and this relationship was specifically due to the targeting of Gag. Furthermore, patients with undetectable viremia had greater Gag-specific responses compared with patients with high viral replication. The most frequently recognized peptides clustered within a defined region of Gag, and responses to a single peptide in this region were associated with low viral burden. The consistent relationship between Gag-specific immune responses and viremia control suggests that $\mathrm{T}$ cell responses are vital in determining the superior outcome of HIV-2 infection. A better understanding of how HIV-2 infection is controlled may identify correlates of effective protective immunity essential for the design of HIV vaccines.
\end{abstract}

\section{Introduction}

HIV infection in humans can be caused by 2 related yet distinct viruses: HIV-1 and HIV-2. Infection with HIV-2 is associated with a reduced rate of progression to AIDS (1) and significantly lower levels of plasma viral RNA $(2,3)$ in spite of similar proviral load (4) and a 30\%-60\% sequence homology between the 2 viruses (5). Consequently, the majority of HIV-2-infected individuals are asymptomatic and die of causes unrelated to immunodeficiency (1). Although HIV-2 infection has no effect on survival in most adults, individuals who do progress to AIDS are clinically indistinguishable from those infected with $\operatorname{HIV}-1(6,7)$, demonstrating that HIV-2 is not simply an attenuated virus.

To date, there is no absolute explanation for the lower viral set point and the long-term nonprogression characteristic of HIV-2 infection. There is evidence to support that control of HIV-2 replication may result from superior immune protection (8-13). In HIV-1 infection, antigen-specific CD8 T cells are important in controlling viremia (14-16), and comprehensive epitope analysis established that virus-specific immune responses can be mounted against the entire HIV-1 proteome (17-23). However, the contribution of the cellular immune system to viremia control is controversial, with evidence to support either a positive $(18,19)$, a negative $(14,24-26)$, or no correlation $(17,21,22)$ with HIV-1 plasma viral load (VL). Previous studies in HIV-2 infection similarly report positive (27), negative $(10,11,28)$, or no correlation $(29,30)$ with clinical markers of disease progression. However, past investigations of HIV-2-specific immune responses have been constrained

Nonstandard abbreviations used: ELISpot, enzyme-linked immunosorbent spot (assay); SFU, spot-forming unit(s); VL, viral load.

Conflict of interest: The authors have declared that no conflict of interest exists. Citation for this article: J. Clin. Invest. 117:3067-3074 (2007). doi:10.1172/JCI32380. by incomplete coverage of the entire HIV-2 proteome, and until now, there has been no comprehensive study of antigen-specific immune responses directed against the HIV-2-expressed genome.

The highest HIV-2 prevalence is in the Republic of Guinea-Bissau, reaching up to $20 \%$ in individuals over 40 years of age (31). In 1989 , the only community-based HIV-2 cohort in the world was established in Caio, a remote village in the northwestern part of the country. Prior studies that examined the role of the immune system in HIV-2 infection have been conducted on clinical cohorts; thus much of what is known about HIV-2-specific immune responses is derived from the minority of HIV-2-positive patients who progress to AIDS and may not be informative of protective immune responses. To date, the contribution of antigen-specific immune responses to HIV-2 viremia control has not been defined, and the Caio cohort provides an excellent opportunity to address this question.

Using an ex vivo IFN- $\gamma$ enzyme-linked immunosorbent spot (ELISpot) assay and a 3-dimensional matrix of overlapping peptides spanning the HIV-2 proteome, we studied the in vivo frequency, relative dominance, breadth, and specificity of HIV-2-specific T cell responses. A better understanding of immune control in HIV-2 infection could reveal the reasons for the attenuated disease course in many infected people and may help to identify correlates of effective protective immunity essential in the design of HIV vaccines.

\section{Results}

Characteristics of participants. Fifty percent of the study participants have been HIV-2 seropositive for at least 17 years (first serological HIV-2 diagnosis made in 1989; ref. 32), and HIV-2 plasma VL did not differ in patients diagnosed before or after 1989, implying this cohort represents a unique population of HIV-infected longterm nonprogressors. Clinical parameters of study participants are displayed in Table 1. 


\section{Table 1}

Clinical and demographic characteristics of study subjects

\begin{tabular}{lcccc} 
& All patients $(\boldsymbol{n}=\mathbf{6 4})$ & $\mathrm{VL}<\mathbf{1 0 0}(\boldsymbol{n}=\mathbf{3 1})$ & VL $\geq \mathbf{1 0 0}(\boldsymbol{n}=\mathbf{3 3})$ & $\boldsymbol{P}$ value \\
HIV-2 VL & $112(<100-283,542)^{\mathrm{A}}$ & $<100$ & $813(109-283,542)^{\mathrm{A}}$ & $\mathrm{NA}$ \\
Absolute CD4 count & $617(140-1,705)^{\mathrm{B}}$ & $713(140-1,705)^{\mathrm{B}}$ & $526(140-1,120)^{\mathrm{B}}$ & 0.014 \\
CD4 percentage & $32.7(13-55)^{\mathrm{B}}$ & $36.5(15-55)^{\mathrm{B}}$ & $29.1(13-45)^{\mathrm{B}}$ & 0.002 \\
Age & $58.2(24-86)^{\mathrm{B}}$ & $55.2(24-86)^{\mathrm{B}}$ & $61.1(27-84)^{\mathrm{B}}$ & 0.110 \\
HIV before 1989 (\%) & 50 & 48.4 & 51.5 & 0.802 \\
Female (\%) & $69 \%$ & $80 \%$ & $58 \%$ & 0.047 \\
\hline
\end{tabular}

AMedian values and range. BMean values and range.

Previous studies that examined HIV-2 VL as a predictor of survival found that HIV-2 RNA plasma levels below the limit of detection ( $<100$ copies/ml) predict normal survival (2) and may be a surrogate marker for long-term nonprogression. Therefore, in this cross-sectional study, we stratified the cohort into 2 groups on the grounds that VL was undetectable (VL $<100$ copies/ml, $n=31$ ) or detectable (VL $\geq 100$ copies/ml, $n=33$ ). This division showed that patients with undetectable HIV-2 VL had higher mean absolute CD4 counts and CD4 percentages; thus, the stratification facilitated studying the relationship between immune response specificity and viremia control in patients with an intact immune system compared with those progressing to AIDS. The overall results of the ELISpot parameters investigated in the patient groups classified by plasma VL are summarized in Table 2 .

Gag is the most frequently recognized HIV-2 gene product, and Nefresponses are infrequent. Using the ELISpot assay, all HIV-2 gene products could stimulate IFN- $\gamma$ secretion after in vitro stimulation with overlapping peptide pools (Figure 1). The majority of HIV-2-infected individuals mounted an HIV-2-specific immune response, with $61 / 64$ patients recognizing at least 1 gene product. The most frequently recognized peptides were in the Gag region of the proteome ( $87.5 \%$ of patients) followed by Env (51.6\% of patients), Pol (43.8\% of patients), Nef (10.9\% of patients), and accessory proteins ( $7.8 \%$ of patients). Within the Gag region (first matrix dimension, pools 1-4), pools 2 and 3 had the highest frequency of positive responses, with $76 \%$ patients exhibiting pool 2 -specific and $68 \%$ patients exhibiting pool 3-specific IFN- $\gamma$ immune responses (data not shown).

Inverse correlation between IFN- $\gamma$ magnitude and HIV-2 VL. The IFN- $\gamma$ responses to the first ELISpot matrix dimension were used to quantify the total number of functional antigen-specific T cells. When the IFN- $\gamma$ immune response to the entire HIV-2 proteome (pools 1-24) was examined in relation to plasma $\mathrm{VL}$, a significant negative correlation was found (Figure 2A). More specifically, this relationship was due to immune responses targeted to the Gag region (70 peptides present in pools 1-4) of the proteome (Figure 2B).

The cohort was next divided into 2 groups according to HIV-2 plasma VL (VL < 100 and VL $\geq 100$ copies $/ \mathrm{ml}$ ) to evaluate the role of the strength of gene product-specific IFN- $\gamma$ immune responses in the control of plasma viremia (Figure $2 \mathrm{C}$ ). The analysis revealed a significantly greater IFN- $\gamma$ immune response magnitude to the proteome and to Gag in subjects with undetectable VL. This relationship was significant only for immune responses targeted to peptides present in pool 2 (500 versus 210 spot-forming units $[\mathrm{SFU}] / 10^{6}$ PBMCs; $P=0.02$ ) and pool 3 (280 versus $85 \mathrm{SFU} / 10^{6}$ PBMCs; $P=0.005)$. No difference was observed between responses targeting other HIV-2 gene products or other peptide pools and the control of viral replication.
After having identified an inverse association between Gag-specific immune responses in patients with and without detectable viremia, we dichotomized the cohort based on plasma VL into the top tenth percentile representing progressing patients (mean VL of 82,647 copies/ $\mathrm{ml}$, range of 9,659-283,542 copies/ $\mathrm{ml}, n=6)$ and undetectable viremia representing normal survival $(<100$ copies $/ \mathrm{ml}$, $n=31$ ). We performed the same analysis on these 2 patient groups to determine if the association observed in the division of the cohort based on VL $<100$ or VL $\geq 100$ copies/ml would be preserved. We found that the magnitude of IFN- $\gamma$ immune responses to the proteome and to Gag was significantly greater in patients with lower VLs $(P=0.0007$ and $P=0.0013$, respectively) and that there was no difference in the magnitude of the other protein-specific immune responses.

Inverse correlation between relative dominance of Gag-specific immune responses and HIV-2 VL. The strength of a protein-specific immune response as a proportion of the entire virus-specific response is defined as the relative dominance (protein-specific IFN- $\gamma$ magnitude/ proteome IFN- $\gamma$ magnitude, $\times 100)$. Analysis of relative dominance identified a hierarchy in HIV-2 protein recognition, with Gag-specific IFN- $\gamma$ secretion dominating the immune response (66\%), followed by Env (16.3\%), Pol (13.7\%), Nef (2.3\%), and accessory proteins (1.7\%). The relative dominance of Gag-specific $\mathrm{T}$ cell responses is particularly striking considering that Gag peptides constituted only $16.5 \%$ of the total peptides used in the ELISpot matrix (Table 3).

\section{Table 2}

Summary of ELISpot responses

$$
\mathrm{VL}<100(n=31) \quad \mathrm{VL} \geq 100(n=33) \quad P \text { value }
$$

IFN- $\gamma$ magnitude ${ }^{A}$

$\begin{array}{lccc}\text { Proteome } & 1,594(95,16,670) & 675(0,6,660) & 0.032 \\ \text { Gag } & 1,120(0,9,640) & 385(0,2,470) & 0.004 \\ \text { Pol } & 0(0,3,953) & 0(0,4,940) & 0.303 \\ \text { Accessory } & 0(0,2,505) & 0(0,1,540) & 0.153 \\ \text { Env } & 130(0,2,480) & 0(0,1,140) & 0.269 \\ \text { Nef } & 0(0,810) & 0(0,465) & 0.719 \\ \text { Relative dominance }(\%) & & \\ \text { Gag } & 88(0,100) & 64.5(0,100) & 0.023 \\ \text { Pol } & 0(0,36) & 6(0,100) & 0.056 \\ \text { Accessory } & 0(0,36) & 0(0,23) & 0.187 \\ \text { Env } & 5(0,61) & 0(4,100) & 0.958 \\ \text { Nef } & 0(0,38) & 0(0,35) & 0.620 \\ \text { BreadthB } & 4.51( \pm 2.65) & 3.76( \pm 2.67) & 0.137\end{array}$

ASFU per $10^{6}$ PBMCs. ${ }^{B}$ Average number of pools recognized per matrix dimension (range 0-24). 


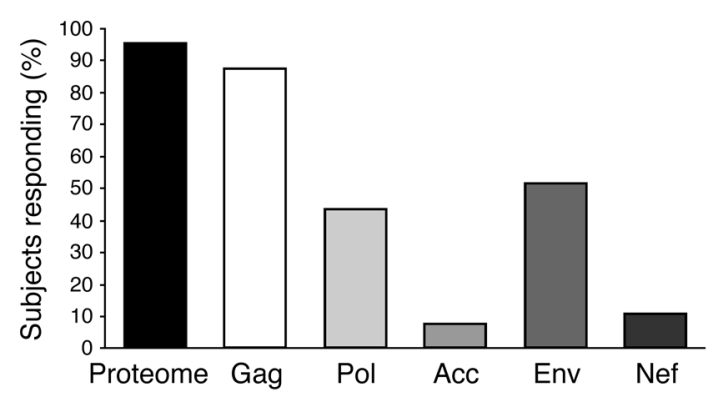

When the cohort was stratified according to plasma VL (Figure 3), the median relative dominance of Gag-specific immune responses was significantly higher in patients who demonstrated control of viremia ( $88 \%$ versus $64.5 \% ; P=0.02$ ). For individuals with $\mathrm{VL} \geq 100$ copies/ml, Pol-specific immune responses tended to contribute more to the total IFN- $\gamma$ HIV-2-specific immune response; however, this difference did not reach statistical significance.

We were further interested in whether the relative dominance of immune responses could be more specifically mapped to individual matrix pools. When the magnitude of each peptide pool was expressed as a proportion of the total proteome IFN- $\gamma$ immune response, only the relative dominance of pool 3-specific responses was associated with HIV-2 VL control ( $P=0.04$; data not shown).

No relationship between the breadth of HIV-2-specific immune responses and control of viremia. To assess whether the breadth of $\mathrm{T}$ cell responses contributes to control of viremia, the average number of matrix pools with positive ELISpot responses was calculated by

\section{Figure 1}

Proportion of patients with positive IFN- $\gamma$ ELISpot responses to HIV-2 gene products. Acc, accessory proteins: Vif, Vpx, Vpr, Tat, Rev.

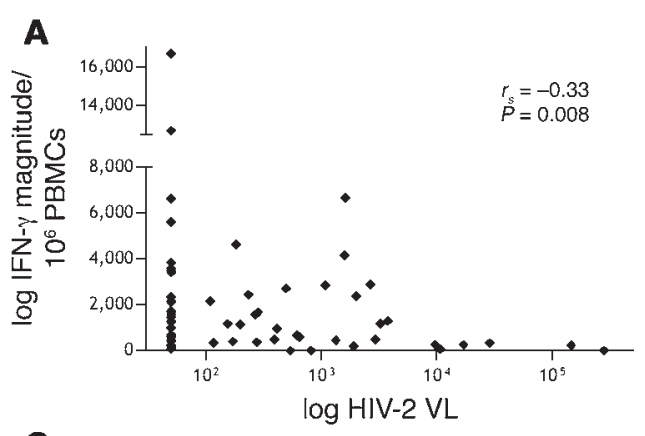

C

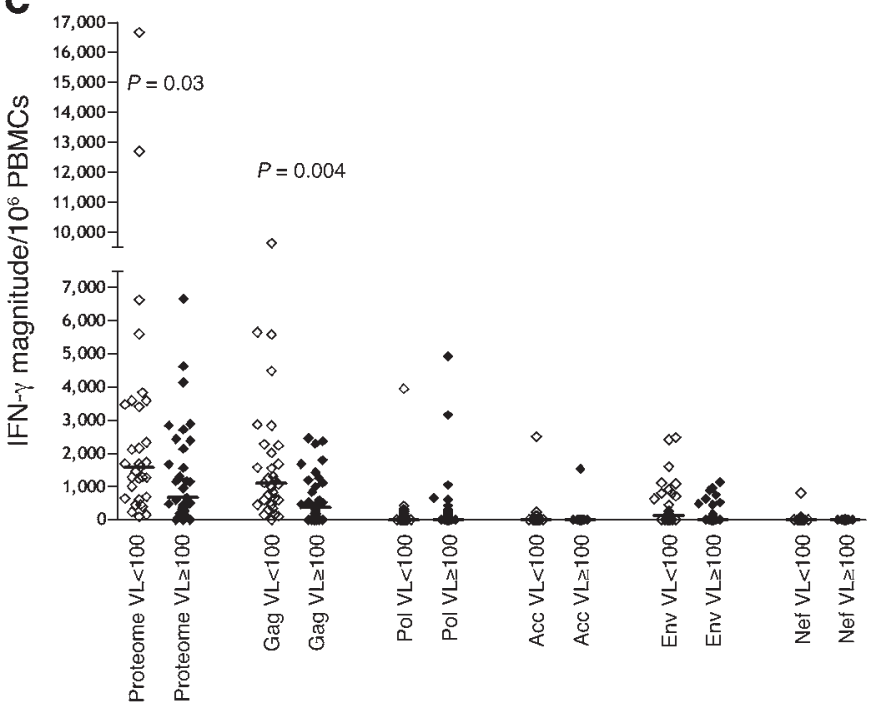

dividing the total number of positive peptide pools by the number of dimensions used in the ELISpot assay (range 0-24 pools). When the average number of positive peptide pools was related to HIV-2 VL (Figure 4A), there was a weak but not significant inverse association between the number of positive pools and plasma VL. Similarly, when the breadth of immune responses was compared among patients with VL $<100$ and $\mathrm{VL} \geq 100$ copies/ml, no difference was detected (Figure 4B).

Most frequently targeted peptides cluster in a narrow part of the virus. The design of the ELISpot matrix allowed us to delineate the precise antigen specificity of HIV-2-specific T cells. We identified 133 epitope-containing peptides, and six of these peptides were recognized by at least $10 \%$ of the cohort (Table 4 ). These 6 peptides clustered in a highly conserved, 149 amino acid-long sequence within the Gag region of HIV-2 proteome ( $\left.\mathrm{Gag}_{175-323}\right)$. This region contained a total of 18 overlapping peptides ( $4.2 \%$ of all of the tested peptides), 13 of which were recognized by at least one subject.

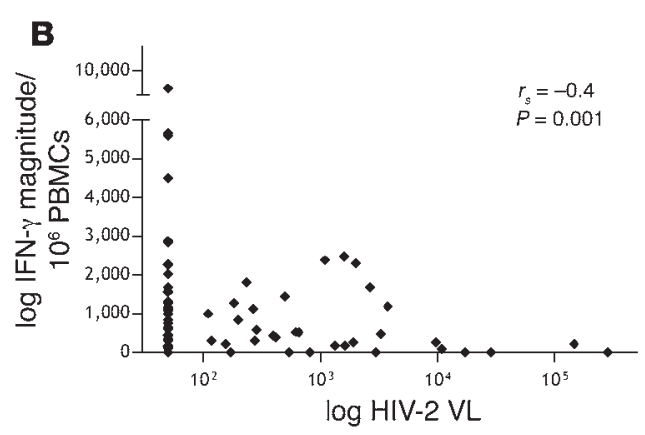

Figure 2

Relationship between HIV-2specific IFN- $\gamma$ immune response magnitude and HIV-2 plasma VL. Correlation between $(\mathbf{A})$ proteome and $(\mathbf{B})$ Gag-specific immune responses and VL (Spearman's rank correlation). Proteome magnitude is the sum of responses in pools 1-24, and Gag magnitude is the sum of responses to pools $1-4$ in the first matrix dimension. (C) Comparison of IFN- $\gamma$ ELISpot response magnitude in patients with undetectable (open diamonds) and detectable (filled diamonds) HIV-2 VL. Black horizontal bars represent median values, and significant $P$ values are indicated. $r_{s}$, Spearman's rank correlation coefficient. 


\section{Table 3}

Distribution of HIV-2 overlapping peptides used in ELISpot matrix

\begin{tabular}{|c|c|c|c|c|c|c|}
\hline Gene product & Gag & Pol & $\operatorname{Acc}^{A}$ & Env & Nef & Total \\
\hline Number of peptides & 70 & 133 & 81 & 107 & 33 & 424 \\
\hline Number of amino acids & 521 & 1,035 & 668 & 862 & 257 & 3,343 \\
\hline Total antigens $(\%)^{\mathrm{B}}$ & 16.5 & 31.4 & 19.1 & 25.2 & 7.8 & 100 \\
\hline Matrix peptide number & $001-077$ & $081-219$ & $221-318$ & $321-438$ & $441-476$ & $001-476$ \\
\hline Matrix pools (first dimension) & $1-4$ & $5-11$ & $12-16$ & $17-22$ & $23-24$ & $1-24$ \\
\hline Average number peptides/pool & 17.5 & 19.0 & 16.2 & 17.8 & 16.5 & 17.7 \\
\hline
\end{tabular}

AAccessory proteins: Vif, Vpx, Vpr, Tat, Rev. BOverlapping peptides encoded by the gene product expressed as percentage of all peptides.

Responses to a single peptide correlate with viremia control. The most frequently recognized peptide was peptide 46 (Gag G $_{28}-315$, YVDRFYKSLRAEQTDPAV), and a response to this peptide was present in $31 \%$ of the cohort. When patients were stratified into either responders or nonresponders to 1 of the 6 most frequently targeted peptides, only the presence of peptide 46-specific responses was inversely related to HIV-2 plasma VL (Figure 5A).

To further characterize peptide 46-specific T cells, we used CD8 ${ }^{+}$ and CD8- PBMC fractions in an IFN- $\gamma$ ELISpot assay and IFN- $\gamma$ intracellular staining to determine $\mathrm{T}$ cell-subtype restriction of these responses. Results showed that peptide 46 responses can be CD 4 and CD8 $\mathrm{T}$ cell restricted (data not shown). We next employed antiHLA-DR antibodies in an IFN- $\gamma$ ELISpot assay in the presence of fresh PBMCs from a peptide 46 responder stimulated with peptide 46. This eliminated the antigen-specific response (Figure 5B), indicating that peptide 46 responses can be restricted by HLA-DR, thus supporting the potential role of CD4 $\mathrm{T}$ cells in the restriction of this epitope-containing peptide.

\section{Discussion}

HIV-2 infection is a relatively neglected model of naturally attenuated HIV infection, with infected subjects falling into 2 broad groups: progressors, who are clinically indistinguishable from people infected with HIV-1, and nonprogressors. As the majority of HIV-2-positive patients have a low or undetectable VL and are long-term nonprogressors, this infection serves as a human model for controlled retroviral infection. The question of what antigen-specific immune responses could account for long-term nonprogression in HIV-2 infection has never been addressed. A more complete understanding of the interplay between the immune system and HIV-2 replication may therefore provide information about correlates of immune protection pertinent to HIV-1 infection. This has been difficult to acquire as nearly all HIV-1 patients progress to disease.

This study, which included 64 patients from a well-characterized community-based cohort with follow-up exceeding 17 years, is, to our knowledge, the largest study to date examining HIV-2-specific T cells and the only study to do so outside clinical cohorts that are dominated by the minority of HIV-2-positive patients who progress to AIDS. We believe it is also the first comprehensive characterization of cellular immune responses against the entire HIV-2 proteome using a novel 3D peptide matrix in an ex vivo IFN- $\gamma$ ELISpot assay. Using this approach, we demonstrated that antigen-specific $\mathrm{T}$ cell responses can be mounted to all HIV-2 proteins. HIV-2-specific immune responses were unevenly distributed across the expressed genome, which is similar to HIV-1-specific responses $(17,18,20,33)$. However, unlike HIV-1 infection, we have identified an unquestionably clear relationship between control of HIV-2 viremia and antigenspecific immune responses to the whole proteome, to Gag, and to a single peptide within the 26 region of Gag.

It is striking that the strongest immune responses were shown significantly more often by individuals with plasma VLs below the limit of assay detection (<100 RNA copies/ml), with HIV-2specific T cells accounting for up to $1.6 \%$ of circulating PBMCs. Interestingly, the most frequently targeted peptides clustered in a highly conserved, 149 amino acid-long region of HIV-2 Gag, representing $28.6 \%$ of the Gag gene product and only $4.5 \%$ of the total HIV-2 proteome. In addition, Gag elicited the most vigorous IFN- $\gamma$ immune responses, reaching up to $9,640 \mathrm{SFU} / 10^{6}$ PBMCs. This suggests that as much as $1 \%$ of the PBMCs of an HIV-2-infected individual can recognize a very narrow part of the viral proteome. Results from stratifying the cohort based on the highest (tenth percentile of all plasma VL) and undetectable VL similarly identified an unequivocal strong negative association between the level of viremia and Gag-specific immune responses, suggesting that the relationship is valid over a wide range of VLs. The importance of Gag recognition became apparent upon matrix deconvolution, which identified a peptide rec-

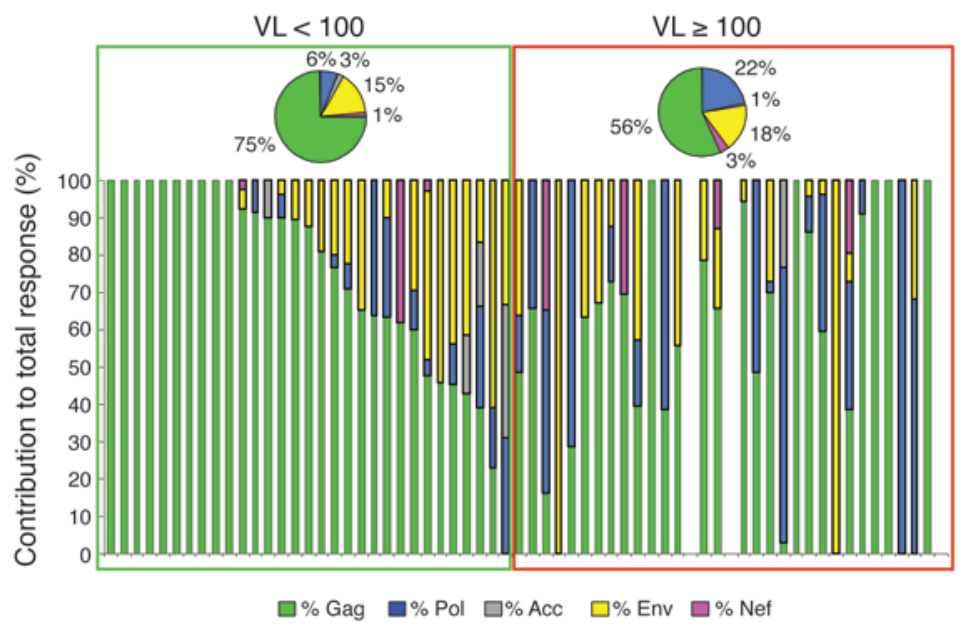

Figure 3

Relative dominance of each gene product for every member of the 2 groups (plasma VL $<100$ or $\geq 100$ copies $/ \mathrm{ml}$ ). Pie charts display mean relative dominance values for each gene product expressed as a percentage of the total IFN- $\gamma$ immune response. 

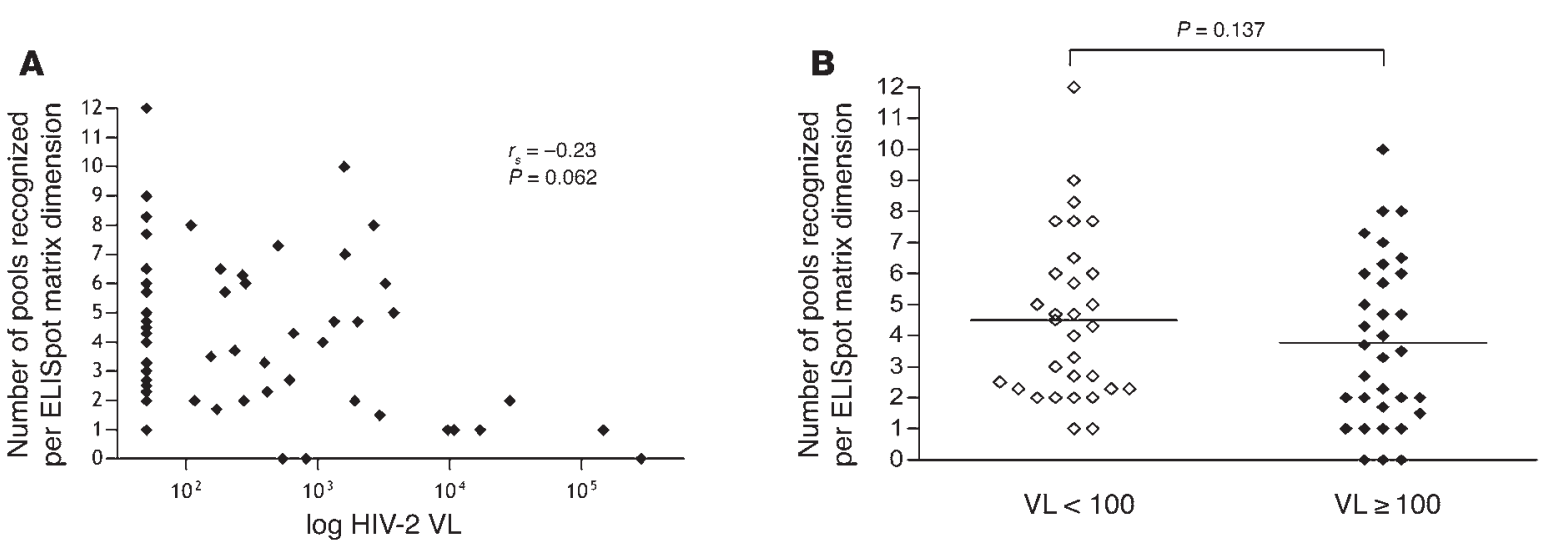

\section{Figure 4}

Relationship between breadth of ELISpot responses and HIV-2 VL. Breadth of ELISpot responses was defined as the average number of pools recognized per matrix dimension (range 0-24). (A) Relationship between the breadth of ELISpot responses and HIV-2 VL. (B) Comparison of breadth of ELISpot responses in patients with controlled viremia (VL $<100 \mathrm{copies} / \mathrm{ml}$; open diamonds) and detectable VL (VL $\geq 100$ copies/ml; filled diamonds).

ognized by over $30 \%$ of the cohort (peptide $46, \mathrm{Gag}_{298-315}$ ), with patients who made responses to this peptide having a lower VL than those who did not.

In HIV-1 infection, Gag is among the most immunogenic regions of the virus (20), and preferential targeting of Gag (18), especially by CD8 T cells $(33,34)$, is associated with enhanced control of viral replication. The region of HIV-1 Gag corresponding to peptide 46 contributes to the total immune response in HIV-1 infection in terms of frequency of recognition (35), immunodominance $(18,36)$, and avidity (22). This frequently targeted and highly immunogenic region of Gag can be restricted by both CD4 $(21,37)$ and CD8 (35) $\mathrm{T}$ cells as well as by a variety of HLA haplotypes (38). However, to our knowledge, this is the first time that this region has been shown to be associated with control of viral replication.

The region of the virus that contains peptide 46 ( $\left.\mathrm{Gag}_{298-315}\right)$ warrants further discussion. Gag g $_{288-315}$ in HIV-2 is located in an area of the virus equivalent to the HIV-1 capsid protein. More precisely, the 18 amino acid-long sequence of the peptide overlaps with the major homology region, a conserved stretch of 20 amino acids found in the carboxylterminal domain of the capsid protein. The major homology region is highly conserved across all retroviruses and is essential for viral assembly, maturation, and infectivity, and its deletion impairs membrane binding, viral particle formation, and correct assembly of the viral core (39-41). In our cohort, analysis of HIV-2 p26 sequences revealed a $96.5 \%$ amino acid sequence identity (Clayton Onyango, unpublished observations). There- fore, evasion of capsid-specific $\mathrm{T}$ cell responses by the virus may be severely limited by the structural and functional requirements imposed on this region $(42,43)$.

A vital aim of HIV vaccine design is eliciting antigen-specific CD8 $\mathrm{T}$ cell responses; thus, it is important that vaccine constructs include areas of the viral genome that are often recognized by antigen-specific T cells, are restricted by various HLA alleles, and are resistant to escape mutations. In naturally infected HIV-1 and HIV-2 subjects, the capsid region of Gag represents such an area, and since peptide 46 is located in this region, it is crucial to further examine the function and phenotype of peptide 46-specific T cells in the context of natural infection and to include this peptide in future CTL-inducing vaccines.

Our comprehensive analysis of HIV-2-specific immune responses demonstrated that a narrow part of the proteome is most often targeted by functional, IFN- $\gamma$-producing T cells. This raises the question of why the host immune system targets the capsid region of the virus so frequently. The capsid region of Gag may be better processed by the host and more effectively presented on MHC molecules relative to other areas of the viral proteome. If this is the case, it would support the prior finding that CTL effectiveness critically depends upon epitope density (44). It is also possible that antigens from this region may be more available for presentation since capsid is expressed at higher levels compared with other parts of viral genome (45). Also, the ample capsid from incoming virions may provide abundant substrate for antigen processing without requiring de novo protein syn-

\section{Table 4}

Most frequently recognized HIV-2 epitope-containing peptides

\begin{tabular}{|c|c|c|c|c|}
\hline Matrix peptide no. & Gene product & Sequence & Frequency ${ }^{A}$ & Average SFU/10 6 PBMCs \\
\hline 46 & $\operatorname{Gag}_{298-315}$ & YVDRFYKSLRAEQTDPAV & 20 & 706 \\
\hline 27 & $\operatorname{Gag}_{175-190}$ & QALSEGCTPYDINQML & 13 & 982 \\
\hline 38 & $\mathrm{Gag}_{251-268}$ & MYRQQNPVPVGNIYRRWI & 11 & 1,403 \\
\hline 47 & $\operatorname{Gag}_{306-323}$ & LRAEQTDPAVKNWMTQTL & 10 & 1,359 \\
\hline 36 & $\operatorname{Gag}_{245-252}$ & SDIAGTTSTVDEQIQWMY & 10 & 662 \\
\hline 41 & $\mathrm{Gag}_{259-276}$ & PVGNIYRRWIQIGLQKCV & 9 & 254 \\
\hline
\end{tabular}

ANumber of patients showing peptide-specific response. 

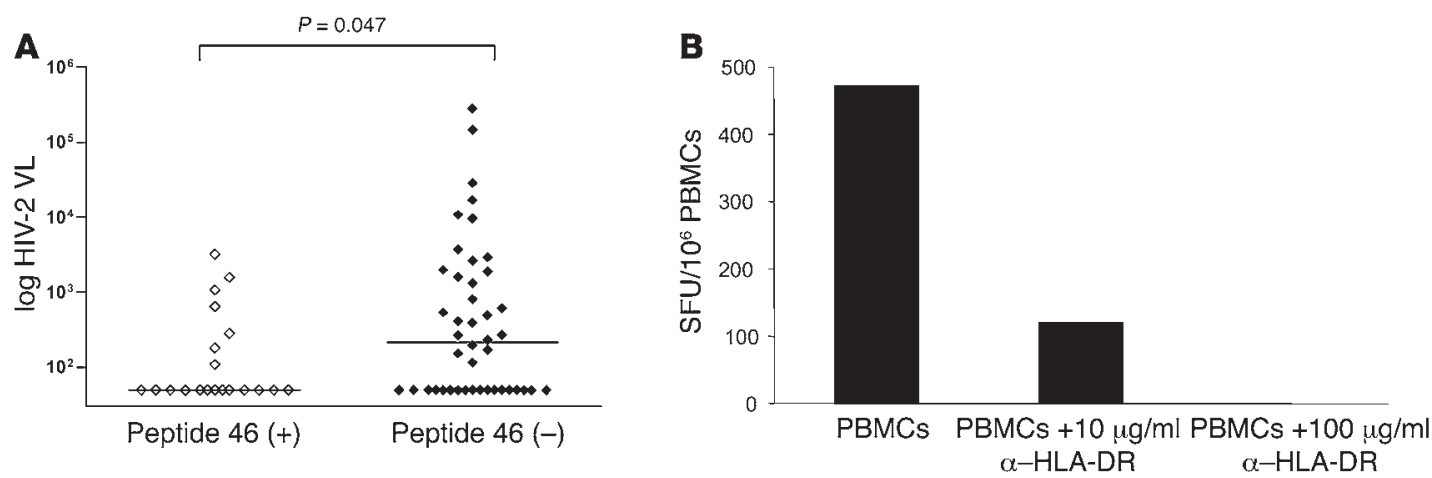

Figure 5

Peptide 46-specific immune responses. (A) Association between peptide 46-specific IFN- $\gamma$ responses and viremia control. Peptide 46 (+) indicates responders (open diamonds; $n=20$ ), and peptide $46(-)$ indicates nonresponders (filled diamonds; $n=44$ ) to this peptide. (B) Peptide 46-specific IFN- $\gamma$ responses can be blocked with anti-HLA-DR antibodies.

thesis early after viral entry (46). Regardless, the capsid area of HIV-1 and HIV-2 Gag represents an equilibrium between the host immune response and the virus functional constraints.

Immunodominance hierarchy of HIV-1 epitopes is determined early in infection and is seldom modified in chronic infection (23). Thus, it is likely that responses to conserved parts of the genome, such as the capsid region of HIV-2 Gag, are made early in the course of infection and are maintained for many years after infection. To address the question of maintenance of antigen-specific responses over time, we first examined individual peptide responses after 6-12 months. We found that responses observed in the initial screen were preserved in the follow-up sample collection. We then proceeded to test to determine whether the length of infection influences the specificity of immune responses. The cohort was stratified according to time of first diagnosis into patients diagnosed before 1989 and infected for over 17 years $(n=32)$ and those diagnosed after 1989 and infected for 3-15 years, median 9 years $(n=32)$. We found that in the 2 subject groups with established HIV-2 infection, there was no difference in the frequency or the relative dominance of Gag-specific immune responses. The detection of robust immune responses to the same region of the virus in many patients irrespective of the duration of infection implies that T cells that target epitopes in areas of the HIV-2 proteome incapable of escaping immune pressure are durable. This is particularly striking considering the high magnitude of responses that appear to have been maintained over decades despite low levels of viral replication. Even though the virus is undetectable in the plasma of the majority of HIV-2-infected individuals, controlled viral replication is likely ongoing in lymphoreticular tissues, thus satisfying the requirement for antigen presence for the maintenance of antigen-specific memory $\mathrm{T}$ cells despite the absence of the virus in the blood. During states of high viremia, the lower frequency of antigen-specific T cells could reflect their exhaustion or their decay, as the high viral replication rate drives viral evasion of the immune response. Taken together, the data suggest that IFN- $\gamma$ immune responses to conserved antigens contribute to the control of HIV-2 viremia and that the resulting low levels of circulating virus may prevent immune exhaustion.

The Gag and Nef proteins are consistently identified as the most frequently targeted parts of the HIV-1 proteome, and responses to Nef contribute to almost one-third of the response to the expressed genome $(17,18,20)$. Therefore, the paucity of HIV-2 Nef-specific immune responses observed in this study deserves comment. In fact, the only difference in HIV-1 and HIV-2 immune response specificity is in the recognition of $\mathrm{Nef}(29)$. To rule out the possibility that the sequence of peptides used in our study did not accurately represent the Nef sequences of circulating viral strains, Nef sequences generated from HIV-2-infected members of the cohort (Jerome Feldmann, unpublished observations) were compared with the Nef consensus sequence used for the synthesis of overlapping peptides. Sequence alignment revealed a mean $82.5 \%$ amino acid identity for the whole protein. When the region of HIV-2 Nef corresponding to the immunodominant part of HIV-1 Nef $\left(\mathrm{Nef}_{70-94}\right)(17)$ was analyzed, an $87 \%$ identity was seen, suggesting that the peptide sequence was a reasonable representation of the HIV-2 Nef sequences present in the community and should have elicited ELISpot responses. There are differences in sequence, size, and function between HIV-1 and HIV-2 Nef proteins. In comparison with HIV-1, HIV-2 Nef is longer (257 aa and $34 \mathrm{kDa}$ versus 208 aa and $27 \mathrm{kDa}$ ), and the sequence homology between the 2 viral consensus sequences has only a 30.9\% identity (Los Alamos National Laboratory HIV Database; http://hiv-web. lanl.gov). It is possible the sequence variation could affect a combination of epitope processing, MHC binding and epitope presentation, or T cell receptor recognition of the peptide-MHC complex, thereby altering HIV-2 Nef-specific immune responses. An alternate possibility is that the level of Nef in controlled HIV-2 infection is insufficient for adequate immunogenicity.

Even though fine specificity of the immune response may account for the distinct clinical outcome associated with long-term nonprogression characteristic of HIV-2 infection and provides in vivo support for the importance of cellular immune responses in the control of viral replication, limitations to this conclusion deserve mention. While IFN- $\gamma$ secretion is antigen specific, it is not the only cytokine secreted upon antigen exposure. Therefore, we are conducting studies to determine the functional profile of antigen-specific cells identified during the cross-sectional analysis of HIV-2-specific immune responses. The IFN- $\gamma$ ELISpot assay does not identify the subset of lymphocytes that contribute to the antigen-specific cytokine secretion. We therefore used IFN- $\gamma$ intracellular staining using flow cytometry to deduce whether CD4 or CD8 T cells were responsible for the cellular immune response. We found that the majority of responses were CD8 T 
cell restricted, suggesting that it is the CD8 subset of $\mathrm{T}$ cells that contributes to HIV-2-specific immune response.

In summary, we have shown that in HIV-2 infection, robust IFN- $\gamma$ immune responses made by antigen-specific $\mathrm{T}$ cells are an important determinant of control of viral replication and disease outcome. Furthermore, immune responses mounted to a narrow and highly conserved region of the HIV-2 capsid region of Gag are a strong marker for control of HIV-2 viremia. We have demonstrated that both the recognition of this region and the magnitude of the IFN- $\gamma$ response mounted by antigen-specific $\mathrm{T}$ cells significantly correlate with low VLs in HIV-2-infected subjects. Our findings suggest that cellular-mediated immunity is far better preserved in nonprogressive HIV-2-positive patients, implying that the immune system strives to control chronic HIV-2 infection. However, determining whether the preserved immune system function in HIV-2 infection is responsible for maintaining lower viremia or whether the characteristically low viral levels favor the preservation of the host immune system requires further investigation.

\section{Methods}

Study participants. Sixty-four subjects of Manjako ethnic background chronically infected with HIV-2 were recruited in Caio, Republic of Guinea-Bissau. HIV screening was performed using the Murex ICE HIV-1.2.0 capture enzyme immunoassay (Murex Diagnostics). Reactive sera were confirmed using an immunochromatographic rapid test for viral differentiation (Hexagon HIV; HUMAN GmbH). Weakly positive HIV-1 or HIV-2 tests or dually positive results were tested by a synthetic peptide-based strip method (Peptilav 1-2; Sanofi Diagnostics Pasteur). Indeterminate results were subjected to HIV-1- and HIV-2-specific PCR using nested primers based on long terminal repeat (LTR) regions specific for the respective virus $(7,47)$. Patients with confirmed HIV-1/HIV-2 dual status were excluded from the study. HIV-2 plasma VL was quantified by RT-PCR using specific LTR primers (47). The lower limit of detection was $100 \mathrm{RNA}$ copies/ml, and results below the level of detection were assigned an arbitrary value of 50 copies $/ \mathrm{ml}$. CD4 count analysis was carried out using a manual total wbc count and differential lymphocyte count based on freshly collected blood. CD4 percentage analysis was done using BD MultiTest reagents and MultiSet software (BD Biosciences - Immunocytometry Systems) on whole blood stabilized in a 5:1 ratio with TransFix (Cytomark). CD4 counts were calculated using the following formula: (total wbc) $\times(\%$ lymphocytes $) \times(\% \mathrm{CD} 4)$. A summary of the cohort's clinical parameters is presented in Table 1.

All participants were antiretroviral naive and provided informed consent. Ethical approval was obtained from the Gambian Government/ MRC Ethics Committee, from the Republic of Guinea-Bissau Ministry of Health, and from the Oxford Tropical Research Ethics Committee (OXTREC), United Kingdom.

HIV-2 overlappingpeptides. Four complete HIV-2 subtype A genome sequences from West Africa available on the Los Alamos National Laboratory HIV Database were used to create a consensus sequence for all HIV-2 gene products (Gag, Pol, Vif, Tat, Rev, Vpr, Vpx, Env, Nef). The consensus sequence was used to generate 424 peptides overlapping by 10 amino acids, which were 15 to 19 amino acids in length (PeptGen, Los Alamos National Laboratory HIV Database; http://www.hiv.lanl.gov/content/hiv-db/PEPTGEN/PeptGenSubmitForm.html). Peptides were synthesized by the PEPscreen service from Sigma-Aldrich (70\% purity as determined by MALDI-TOF mass spectrometry). The final ELISpot assay peptide concentration was $2 \mu \mathrm{g} / \mathrm{ml}$ per peptide, and the final DMSO concentration in any pool was always less than or equal to $0.04 \%$. The distribution of overlapping peptides is summarized in Table 3 .

Design of a $3 D$ peptide matrix. Overlapping peptides spanning the HIV-2 proteome were arranged in a $3 \mathrm{D}$ matrix of 24 pools per dimension with
13-20 peptides per pool. This peptide configuration was deduced by Deconvolute This! software (48) as the most optimal matrix design based on the number of expected positive peptides. Since there is no information on HIV-2 specific immune responses to the entire HIV-2 expressed genome, we derived the expected number of positive peptides based on data from HIV-1-specific IFN- $\gamma$ responses that suggest that approximately 18 epitopic regions are recognized (17). Past data show that the mean magnitude of responses to homologous HIV-1 and HIV-2 Gag, Tat, and Env gene products is either similar (29) or lower when compared with HIV-1-specific responses (27). Therefore, recognition of 18 or less epitopic regions would be expected per HIV-2-positive subject.

Peptides in the first matrix dimension (pools 1-24) were arranged in sequential order, allowing the calculation of IFN- $\gamma$ responses to individual gene products (Gag, Pol, accessory proteins, Env, Nef). Total IFN- $\gamma$ immune response to the proteome was calculated by averaging the IFN- $\gamma$ magnitude of each of the 3 dimensions. Peptides in the second and third matrix dimensions were in a predicted random distribution that permitted the identification of epitope-containing peptides. For an epitope-containing peptide to be positive, a response must have been present in a unique 3 -pool pattern ( 1 pool in each of 3 dimensions). Only peptides for which the average SFU of the 3 pools was less than 3 times the standard deviation from the average of the 3 pools were used in the analysis to prevent overestimation of epitope-containing peptides.

Ex vivo IFN- $\gamma$ ELISpot assay. Freshly isolated PBMCs were used in ex vivo IFN- $\gamma$ ELISpot assays. Assays were carried out in 96-well MultiScreen filter plates (Millipore) coated with $15 \mu \mathrm{g} / \mathrm{ml}$ of anti-IFN- $\gamma$ monoclonal antibody (1-DIK, Mabtech). PBMCs were added at $10^{5}$ cells/well in a volume of $80 \mu \mathrm{l}$ of $\mathrm{H} 10$ medium (RPMI 1640 [Sigma-Aldrich], 10\% human AB serum, $2 \mathrm{mM}$ L-glutamine, $50 \mathrm{U} / \mathrm{ml}$ penicillin/streptomycin) and stimulated with $20 \mu \mathrm{l}$ of $10 \mu \mathrm{g} / \mathrm{ml}$ peptide pools (final concentration $2 \mu \mathrm{g} / \mathrm{ml}$ per peptide), $5 \mu \mathrm{g} / \mathrm{ml}$ phytohemagglutinin (final concentration $1 \mu \mathrm{g} / \mathrm{ml}$ ), $10 \mu \mathrm{g} / \mathrm{ml}$ optimized CD8 influenza A, EBV, and CMV (FEC) epitopes (16-20 peptides/pool, final concentration $2 \mu \mathrm{g} / \mathrm{ml}$ ) or media control in quadruplicate. Anti-HLA-DR L243 blocking antibodies (ATCC; LGC Promochem) were used at $10 \mu \mathrm{g} / \mathrm{ml}$ and $100 \mu \mathrm{g} / \mathrm{ml}$ under the same assay conditions. Plates were incubated for 16 hours at $37^{\circ} \mathrm{C}, 5 \% \mathrm{CO}_{2}$. Spot enumeration was performed with an AID ELISpot reader system (Autoimmun Diagnostika $\mathrm{GmbH}$ ). To quantify antigen-specific responses, mean spots of the control wells were subtracted from the positive wells and results were expressed as $\mathrm{SFU} / 10^{6} \mathrm{PBMCs}$. Responses were regarded as positive if results were at least 3 times the mean of the quadruplicate negative control wells and over $50 \mathrm{SFU} / 10^{6}$ PBMCs. If background wells were more than $30 \mathrm{SFU} / 10^{6}$ PBMCs or if both positive control wells (phytohemagglutinin or FEC stimulation) were negative, the assay was excluded from further analysis.

Statistics. Statistical analysis was performed using Stata 8.0 (Stata), and graphical presentation was done using Microsoft Excel 2003 and GraphPad Prism 4.02 (GraphPad Software). Normally distributed or transformed data were analyzed using parametric tests: Pearson's correlation or 2-sample unpaired Student's $t$ tests. Nonnormally distributed data were analyzed using nonparametric tests: Spearman's rank correlation or Mann-Whitney $U$ test. Results are given as means with standard deviations (parametric tests) or medians with ranges (nonparametric tests). Statistical test differences were considered significant if $P$ values were less than 0.05 .

\section{Acknowledgments}

We acknowledge M. Roederer from Vaccine Research Center, National Institute of Allergy and Infectious Diseases, NIH, for the Deconvolute This! software. We thank Medical Research Council staff from The Gambia including Sarah Crozier for advice on statistical analysis; Mamady Njie and Samuel Nyam- 
weya for technical lab support; Jainaba Njie and Gibril Bah for CD4 count analysis; and Kevin Taylor, Jerreh Sanyang, and Oluranti Oyeneye for logistical support. Further thanks extend to the field staff in Caio, Guinea-Bissau, for their expertise in patient recruitment and to staff at the Projecto Saude Bandim in Bissau, Guinea-Bissau. This work was supported by the Medical Research Council, United Kingdom, and the Rhodes Trust (Rhodes Scholarship to A. Leligdowicz).

1. Marlink, R., et al. 1994. Reduced rate of disease development after HIV-2 infection as compared to HIV-1. Science. 265:1587-1590.

2. Berry, N., et al. 2002. Low level viremia and high CD $4 \%$ predict normal survival in a cohort of HIV type-2-infected villagers. AIDS Res. Hum. Retroviruses. 18:1167-1173.

3. Andersson, S., et al. 2000. Plasma viral load in HIV1 and HIV-2 singly and dually infected individuals in Guinea-Bissau, West Africa: significantly lower plasma virus set point in HIV-2 infection than in HIV-1 infection. Arch. Intern. Med. 160:3286-3293.

4. Popper, S.J., et al. 2000. Low plasma human immunodeficiency virus type 2 viral load is independent of proviral load: low virus production in vivo. J. Virol. 74:1554-1557.

5. Guyader, M., et al. 1987. Genome organization and transactivation of the human immunodeficiency virus type 2. Nature. 326:662-669.

6. Whittle, H., et al. 1992. Clinical and laboratory predictors of survival in Gambian patients with symptomatic HIV-1 or HIV-2 infection. AIDS. 6:685-689.

7. Ariyoshi, K., et al. 2000. Plasma RNA viral load predicts the rate of CD4 $\mathrm{T}$ cell decline and death in HIV-2-infected patients in West Africa. AIDS. 14:339-344.

8. Bertoletti, A., et al. 1998. Cytotoxic T cells from human immunodeficiency virus type 2 -infected patients frequently cross-react with different human immunodeficiency virus type 1 clades. J. Virol. 72:2439-2448.

9. Gotch, F., et al. 1993. Cytotoxic T cells in HIV2 seropositive Gambians. Identification of a virus-specific MHC-restricted peptide epitope. J. Immunol. 151:3361-3369.

10. Sarr, A.D., et al. 2001. Robust HIV type 2 cellular immune response measured by a modified anthrax toxin-based enzyme-linked immunospot assay. AIDS Res. Hum. Retroviruses. 17:1257-1264.

11. Duvall, M.G., et al. 2006. Maintenance of HIV-specific CD4+ T cell help distinguishes HIV-2 from HIV-1 infection. J. Immunol. 176:6973-6981.

12. Lopes, A.R., et al. 2003. Greater CD8+ TCR heterogeneity and functional flexibility in HIV-2 compared to HIV-1 infection. J. Immunol. 171:307-316.

13. Rowland-Jones, S., et al. 1995. HIV-specific cytotoxic T-cells in HIV-exposed but uninfected Gambian women. Nat. Med. 1:59-64.

14. Ogg, G.S., et al. 1998. Quantitation of HIV-1-specific cytotoxic $\mathrm{T}$ lymphocytes and plasma load of viral RNA. Science. 279:2103-2106.

15. Walker, B.D., et al. 1987. HIV-specific cytotoxic T lymphocytes in seropositive individuals. Nature. 328:345-348.

16. Borrow, P., Lewicki, H., Hahn, B.H., Shaw, G.M., and Oldstone, M.B. 1994. Virus-specific CD8+ cytotoxic T-lymphocyte activity associated with control of viremia in primary human immunodeficiency virus type 1 infection. J. Virol. 68:6103-6110.

17. Addo, M.M., et al. 2003. Comprehensive epitope analysis of human immunodeficiency virus type 1 (HIV-1)-specific T-cell responses directed against the entire expressed HIV-1 genome demonstrate
Received for publication April 11, 2007, and accepted in revised form June 13, 2007.

Address correspondence to: Aleksandra Leligdowicz, Weatherall Institute of Molecular Medicine, MRC Human Immunology Unit, John Radcliffe Hospital, Oxford, OX3 9DS, United Kingdom. Phone: 44-1865-222312; Fax: 44-1865-222502; E-mail: aleksandra. leligdowicz@balliol.ox.ac.uk. broadly directed responses, but no correlation to viral load. J. Virol. 77:2081-2092.

18. Masemola, A., et al. 2004. Hierarchical targeting of subtype $C$ human immunodeficiency virus type 1 proteins by CD $8+\mathrm{T}$ cells: correlation with viral load. J. Virol. 78:3233-3243.

19. Betts, M.R., et al. 2001. Analysis of total human immunodeficiency virus (HIV)-specific CD $4(+)$ and CD8(+) T-cell responses: relationship to viral load in untreated HIV infection. J. Virol. 75:11983-11991.

20. Novitsky, V., et al. 2002. Magnitude and frequency of cytotoxic T-lymphocyte responses: identification of immunodominant regions of human immunodeficiency virus type 1 subtype C. J. Virol. 76:10155-10168.

21. Kaufmann, D.E., et al. 2004. Comprehensive analysis of human immunodeficiency virus type 1-specific CD4 responses reveals marked immunodominance of gag and nef and the presence of broadly recognized peptides. J. Virol. 78:4463-4477.

22. Cao, J., et al. 2003. Comprehensive analysis of human immunodeficiency virus type 1 (HIV-1)specific gamma interferon-secreting CD8+ T cells in primary HIV-1 infection. J. Virol. 77:6867-6878.

23. Yu, X.G., et al. 2002. Consistent patterns in the development and immunodominance of human immunodeficiency virus type 1 (HIV-1)-specific CD8+ T-cell responses following acute HIV-1 infection. J. Virol. 76:8690-8701.

24. Edwards, B.H., et al. 2002. Magnitude of functional $\mathrm{CD} 8+\mathrm{T}$-cell responses to the gag protein of human immunodeficiency virus type 1 correlates inversely with viral load in plasma. J. Virol. 76:2298-2305.

25. Kalams, S.A., et al. 1999. Association between virus-specific cytotoxic T-lymphocyte and helper responses in human immunodeficiency virus type 1 infection. J. Virol. 73:6715-6720.

26. Rinaldo, C., et al. 1995. High levels of anti-human immunodeficiency virus type 1 (HIV-1) memory cytotoxic T-lymphocyte activity and low viral load are associated with lack of disease in HIV-1-infected long-term nonprogressors. J. Virol. 69:5838-5842.

27. Gillespie, G.M., et al. 2005. CD8+ T cell responses to human immunodeficiency viruses type 2 (HIV-2) and type 1 (HIV-1) gag proteins are distinguishable by magnitude and breadth but not cellular phenotype. Eur. J. Immunol. 35:1445-1453.

28. Ariyoshi, K., et al. 1995. HIV-2-specific cytotoxic Tlymphocyte activity is inversely related to proviral load. AIDS. 9:555-559.

29. Zheng, N.N., et al. 2004. Comparison of human immunodeficiency virus (HIV)-specific T-cell responses in HIV-1- and HIV-2-infected individuals in Senegal. J. Virol. 78:13934-13942.

30. Jaye, A., et al. 2004. No differences in cellular immune responses between asymptomatic HIV type 1 - and type 2 -infected Gambian patients. J. Infect. Dis. 189:498-505.

31. Poulsen, A.G., et al. 1993. HIV-2 infection in Bissau, West Africa, 1987-1989: incidence, prevalences, and routes of transmission. J. Acquir. Immune Defic. Syndr. 6:941-948.

32. Wilkins, A., et al. 1993. The epidemiology of HIV infection in a rural area of Guinea-Bissau. AIDS.
7:1119-1122

33. Ramduth, D., et al. 2005. Differential immunogenicity of HIV-1 clade C proteins in eliciting CD8+ and CD4+ cell responses. J. Infect. Dis. 192: $1588-1596$.

34. Kiepiela, P., et al. 2007. CD8(+) T-cell responses to different HIV proteins have discordant associations with viral load. Nat. Med. 13:46-53.

35. Currier, J.R., et al. 2006. CTL epitope distribution patterns in the Gag and Nef proteins of HIV-1 from subtype A infected subjects in Kenya: use of multiple peptide sets increases the detectable breadth of the CTL response. BMC Immunol. 7:8.

36. Goulder, P.J., et al. 2000. Differential narrow focusing of immunodominant human immunodeficiency virus gag-specific cytotoxic T-lymphocyte responses in infected African and caucasoid adults and children. J. Virol. 74:5679-5690.

37. Malhotra, U., et al. 2001. Role for HLA class II molecules in HIV-1 suppression and cellular immunity following antiretroviral treatment. J. Clin. Invest. 107:505-517.

38. Ogg, G.S., et al. 1998. Four novel cytotoxic T-lymphocyte epitopes in the highly conserved major homology region of HIV-1 Gag, restricted through $\mathrm{B} * 4402, \mathrm{~B} * 1801, \mathrm{~A} * 2601, \mathrm{~B} * 70(\mathrm{~B} * 1509)$. AIDS. 12:1561-1563.

39. Gamble, T.R., et al. 1997. Structure of the carboxylterminal dimerization domain of the HIV-1 capsid protein. Science. 278:849-853.

40. Provitera, P., et al. 2001. Role of the major homology region in assembly of HIV-1 Gag. Biochemistry. 40:5565-5572.

41. Mammano, F., Ohagen, A., Hoglund, S., and Gottlinger, H.G. 1994. Role of the major homology region of human immunodeficiency virus type 1 in virion morphogenesis. J. Virol. 68:4927-4936.

42. Martinez-Picado, J., et al. 2006. Fitness cost of escape mutations in p24 Gag in association with control of human immunodeficiency virus type 1 . J. Virol. 80:3617-3623.

43. Melamed, D., et al. 2004. The conserved carboxy terminus of the capsid domain of human immunodeficiency virus type $1 \mathrm{gag}$ protein is important for virion assembly and release. J. Virol. 78:9675-9688.

44. Tsomides, T.J., et al. 1994. Naturally processed viral peptides recognized by cytotoxic T lymphocytes on cells chronically infected by human immunodeficiency virus type 1. J. Exp. Med. 180:1283-1293.

45. Chertova, E., et al. 2006. Proteomic and biochemical analysis of purified human immunodeficiency virus type 1 produced from infected monocytederived macrophages. J. Virol. 80:9039-9052.

46. Sacha, J.B., et al. 2007. Gag-specific CD8+ T lymphocytes recognize infected cells before AIDS-virus integration and viral protein expression. J. Immunol. 178:2746-2754.

47. Berry, N., et al. 1998. Low peripheral blood viral HIV-2 RNA in individuals with high CD4 percentage differentiates HIV-2 from HIV-1 infection. J. Hum. Virol. 1:457-468.

48. Roederer, M., and Koup, R.A. 2003. Optimized determination of $\mathrm{T}$ cell epitope responses. J. Immunol. Methods. 274:221-228. 